\title{
Structure and dynamics of Langmuir-Blodgett tristearin films: Atomic force microscopy and theoretical analysis
}

\author{
Aneliya N. Zdravkova*, J.P.J.M. van der Eerden \\ Department of Condensed Matter and Interfaces, Utrecht University, PO Box 80000, 3508 TA Utrecht, The Netherlands
}

Received 11 April 2006; received in revised form 19 May 2006; accepted 25 May 2006

Communicated by Y. Furukawa

\begin{abstract}
The structure and temporal evolution of tristearin (SSS) monolayers at the air-water interface at $20 \pm 1{ }^{\circ} \mathrm{C}$ are investigated with the Langmuir method. The deposited Langmuir-Blodgett (LB) layers were investigated with atomic force microscopy (AFM). The LB experiments showed that adsorption isotherms obtained with commonly used compression rates do not correspond to thermodynamic equilibrium. Under isobaric conditions at $\pi \geqslant 10 \mathrm{mN} / \mathrm{m}$, a slow compression was found, corresponding to the formation of crystals on top of the monolayer. The AFM images reveal that SSS initially form trident monolayers at air-water interface. These layers are thermodynamically stable at surface pressure $\pi \leqslant 5 \mathrm{mN} / \mathrm{m}$. The thickness of the trident monolayer was found to be $1.6-1.8 \mathrm{~nm}$, corresponding to tilt angles of the molecule chains varying from $\tau=43^{\circ}$ at $\pi=10 \mathrm{mN} / \mathrm{m}$ to $\tau=53^{\circ}$ at $\pi=40 \mathrm{mN} / \mathrm{m}$. For $\pi \geqslant 10 \mathrm{mN} / \mathrm{m}$, growth of crystals takes place with a tuning fork conformation of the SSS molecules on top of the trident monolayer. The crystals grow with time, mainly in lateral directions. The growth rate increases with surface pressure. A new model is developed to quantitatively describe the crystal growth process. A lateral growth rate of $2.3 \mathrm{~nm} / \mathrm{min}$ and a vertical growth rate of $0.005 \mathrm{~nm} / \mathrm{min}$ were calculated for 1 individual crystal at $\pi=10 \mathrm{mN} / \mathrm{m}$. The same growth process that was observed on the air-water interface was also observed in transferred monolayers at room temperature, though the growth was much slower.
\end{abstract}

(C) 2006 Elsevier B.V. All rights reserved.

PACS: $68.18 ;$ 68.37.Ps; 68.47.Pe

Keywords: A1. Atomic force microscopy; A1. Langmuir-Blodgett technique; A1. Surface structure; B1. Triglycerides

\section{Introduction}

Many efforts have been made in investigating the structure of triglycerides. Most of the published work has been on homogeneous triglycerides (their three fatty acid residues are identical). In the solid state, triglycerides adopt a polymorphic crystalline structure.

Depending on the crystallization procedure, especially the thermal treatment, they may crystallize in the $\alpha$ (hexagonal, less stable), $\beta^{\prime}$ (orthorhombic) or $\beta$ (triclinic, most stable) form. In each of these polymorphic forms, the molecules have a tuning fork conformation [1,2], but the packing of these tuning forks is different.

\footnotetext{
${ }^{*}$ Corresponding author. Fax: + 31302532403.

E-mail address: a.n.zdravkova@phys.uu.nl (A.N. Zdravkova).
}

However, in monolayers at a hydrophilic-hydrophobic interface, triglyceride molecules adopt a trident conformation (all hydrocarbon chains pointing toward the same direction). This conformation has been proposed by Bursh et al. [3], based on their $\pi-\mathrm{A}$ diagrams for triglycerides on water at different temperatures. The trident conformation was also found by Hamilton [4,5], using NMR measurements for tripalmitin and triolein at the oil/water interface in phospholipids vesicles and by Claesson et al. [6] for triolein in contact with mica. In the trident conformation, the hydrophilic glycerol group is in contact with the water or the mica surface, and the hydrophobic chains point into the air or oil.

In some cases, multilayers can be formed, when on an interface a monolayer is compressed laterally [7-9].

Bursh and Larsson investigated what happened when a monolayer of triglyceride at the air-water interface is 
compressed beyond the so-called collapse pressure, where the steady increase of the spreading pressure upon lateral compression is interrupted. They concluded that some molecules leave the monolayer to form new molecular layers. They proposed a trident conformation for the first triglyceride monolayer and a tuning fork conformation in the next layers, with a packing similar to that in the crystalline state [3]. Triple layer formation was reported also for bile acids [10]. Only a few studies of triglycerides with atomic force microscopy (AFM) were performed [11,12]. Michalski et al. [12] investigated LangmuirBlodgett (LB) monolayers on glass of tripalmitin by AFM. The monolayer was compressed and withdrawn at a surface pressure, corresponding to the middle of the condensed phase in the $\pi-\mathrm{A}$ isotherm. She suggested that the trident monolayer generally reorganizes after being transferred to the glass, forming two different structures. The first one corresponds to bilayers in a regular tuning fork crystalline structure. The second one corresponds to the triple layer structure, proposed by Bursh et al. [3].

The aim of this paper is to better understand the molecular structure and processes in triglyceride films at the air-water interface (Langmuir film) and on a solid surface like mica (LB film). Therefore, we measured the $\pi-A$ (spreading pressure $\pi$ vs. area per molecule $A$ ) diagram of Langmuir films and we investigated LB films with AFM. In this work, we focus on tristearin (SSS); in subsequent work, we extend the investigations to other triglycerides. Starting with a Langmuir film at very small $\pi$, where the film is in a low-density "gas" phase, we compressed the film, at a constant rate, to the desired pressure $\pi$ (forced compression). To investigate whether the Langmuir film was in thermodynamic equilibrium at this pressure $\pi$, we sometimes left the film for some time $t$ at pressure $\pi$ (isobaric compression). The Langmuir film was transferred to mica directly after forced compression $(t=0)$ or after $t=30 \mathrm{~min}$ or $60 \mathrm{~min}$ of incubation time at constant pressure $\pi$ (isobaric compression).

\section{Materials and methods}

\subsection{Chemicals}

Film material: Tristearin (1, 2, 3, -trioctadecanoylglycerol: SSS) was purchased from Larodan with a stated purity of $>99$ mass $\%$. A stock solution of SSS with concentration of $1 \mathrm{mM}$ in distilled chloroform was prepared.

Subphase: Distilled water was used as a subphase in our Langmuir system for all experiments. The resistivity of the water was $15 \mathrm{M} \Omega \mathrm{cm}$.

Substrates: All monolayers were transferred onto freshly cleaved mica.

\subsection{Langmuir method}

Compression isotherms were measured on a home-made instrument, using available components. The instrument was equipped with a Teflon trough $(8.6 \times 14.8 \mathrm{~cm})$. The spreading pressure $\pi$ was measured with a Wilhelmy type balance consisting of a platinum plate coupled to an electrobalance (Cahn 1000, Ankersmit), with an accuracy of about $0.1 \mathrm{mN} / \mathrm{m}$. The film material was initially spread on the water subphase, dropping $20 \mu \mathrm{L}$ of $1 \mathrm{mM} \mathrm{SSS}$ dissolved in chloroform, using a $25 \mu \mathrm{L}$ Hamilton syringe. The conditions were chosen such that initially the average area $A$ per molecule is $A \sim 110 \AA^{2}$. We started (asymmetric) film compression 2 min after spreading. In our system, two modes of operation were available. First forced compression, where the position of the barrier, and hence the trough length $l(t)$ ahead of the barrier, is given. Then the resulting spreading pressure $\pi(t)$ is registered. In this mode, we chose barrier velocities of the order of $1 \mathrm{~cm} / \mathrm{min}$, which according to the literature should be slow enough that the Langmuir film stays close to thermodynamic equilibrium.

Second we used the isobaric compression mode, where a constant spreading pressure $\pi$ is applied and the resulting trough length $l(t)$ is monitored. Obviously, if the film is in equilibrium at the applied pressure, then $l(t)$ is constant. In practice, however, we often found the barrier to move with velocities of the order of $1 \mu \mathrm{m} / \mathrm{s}$. This barrier motion reflects rearranging processes in the Langmuir film. We use AFM images to interpret and quantify this process.

\subsection{LB film transfer}

In order to obtain LB films, first a substrate was immersed perpendicularly in the aqueous subphase. We started with a very small initial surface pressure $(\pi=0 \mathrm{mN} / \mathrm{m})$, and compressed the monolayer slowly $(1 \mathrm{~cm} / \mathrm{min})$ to the final pressure. To obtain a LB film that is characteristic for forced compression, the film was then transferred immediately by vertical pulling of the substrate through the air-water interface at a speed of $2 \mathrm{~mm} / \mathrm{min}$. During the transfer, the surface pressure was kept constant by appropriately moving the barrier. The transfer process takes a few minutes.

In order to study the structural changes of the Langmuir film during isobaric compression, the film was left at constant pressure for 30 or $60 \mathrm{~min}$ before it was transferred to the substrate. After deposition, the LB films were dried in air and kept in close containers until use. All experiments were done at $20 \pm 1{ }^{\circ} \mathrm{C}$.

\subsection{AFM measurements}

The samples were examined with AFM within about $5 \mathrm{~h}$ after preparation. We checked that the length of this delay time is not critical. Imaging was done with a Nanoscope $(\mathrm{R})$ IIIa (Digital Instruments, Santa Barbara, CA) in contact mode with oxide-sharpened silicon nitride tip $(k=0.06 \mathrm{~N} / \mathrm{m})$. The AFM was equipped with a $\mathbf{J}$ scanner $(176 \times 176 \mu \mathrm{m} ; z$ limit $=5.349 \mu \mathrm{m})$. All images were processed using procedures for flattening in Nanoscope III software version $5.12 \mathrm{r} 5$ without any filtering. To check if 
the monolayer is successfully transferred to the mica surface, we measured at least five different spots (each $150 \mu \mathrm{m}^{2}$ ) of every sample. In order to detect structural changes in the adsorbed film in contact with air, we studied LB films several days after preparation as well.

\section{Langmuir observations}

\subsection{Forced compression}

Fig. 1 shows a typical $\pi-A$ isotherm of SSS, recorded at a barrier velocity of $1 \mathrm{~cm} / \mathrm{min}$. Three different regimes can be recognized. Starting at a large area per molecule $A$, the pressure is low and increases only slowly with decreasing $A$. Upon decreasing $A$ further, the condensation area $A_{\text {cond }}$ is reached and the pressure starts to increase more rapidly. Compressing further, it is seen that for $A$ below the collapse area $A_{\mathrm{col}}$, the increase of the pressure is slow again. The explanation of this characteristic dependence is that for $A_{\text {cond }}=A=A_{\text {col }}$, the SSS molecules are close enough together to form a condensed monolayer, whereas for $A<A_{\text {col }}$, this monolayer collapses to form multilayer structures. The measured $\pi-A$ data showed that the transition from one regime to another were not very sharp. It order to get reliable and unbiased estimations for $A_{\text {cond }}$, $A_{\mathrm{col}}$ and the collapse pressure $\pi_{\mathrm{col}}$, we fitted the isotherms with:

$$
\begin{aligned}
\pi(A) \approx & \left(s_{\mathrm{col}}-\frac{\pi_{\mathrm{col}}}{A_{\mathrm{col}}-A_{\mathrm{cond}}}\right) h\left(A-A_{\mathrm{col}}, a\right) \\
& +\frac{\pi_{\mathrm{col}}}{A_{\mathrm{col}}-A_{\mathrm{cond}}} h\left(A-A_{\mathrm{cond}}, b\right),
\end{aligned}
$$

where $A_{\text {cond }}, A_{\mathrm{col}}, \pi_{\mathrm{col}}, s_{\mathrm{col}}, a$ and $b$ are fitting parameters, $s_{\mathrm{col}}$ representing the slope of the isotherm during collapse, i.e. for $A<A_{\mathrm{col}}$ and $a$ and $b$ characterize the smoothness of

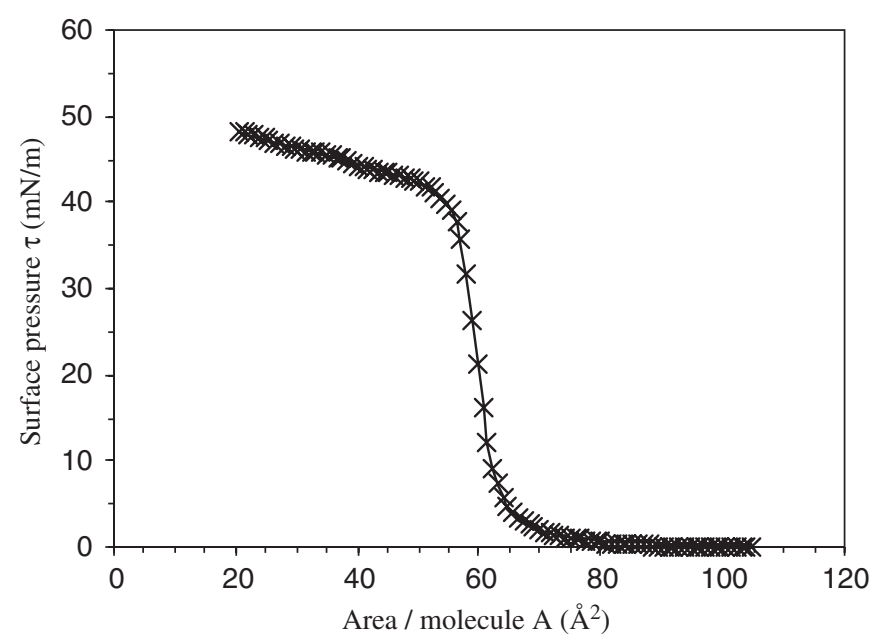

Fig. 1. Example of surface pressure vs. area isotherm of tristearin (SSS) at air-water interface, at $20^{\circ} \mathrm{C}$, obtained by forced compression at a rate of $1 \mathrm{~cm} / \min$ ( $x$ - observed data; - - fit using Eq. (1) with $A_{\text {cond }}=604 \AA^{2}, A_{\text {col }}=$ $58 \AA^{2}$ and $\pi_{\text {col }}=40.5 \mathrm{mN} / \mathrm{m}$. the transitions from one regime to the other. The function

$h(x, a) \equiv \frac{1}{2}\left(x-\sqrt{x^{2}+a^{2}}\right)$

is a hyperbola interpolating between $h(x, a) \approx x$ for large negative $x$ and $h(x, a) \approx 0$ for large positive $x$. This function has no direct physical interpretation and was introduced for practical purposes only. As shown in Fig. 1, satisfactory fits were obtained. Fitting a number of isotherms that were obtained at compression velocities varying from 0.5 to $2 \mathrm{~cm} / \mathrm{min}$, we found $A_{\text {cond }}=62 \pm 2 \AA^{2}, A_{\text {col }}=57.8 \pm 0.3 \AA$ and $\pi_{\mathrm{col}}=41 \pm 1 \mathrm{mN} / \mathrm{m}$. These values did not vary significantly within the range of the barrier velocities that we applied. The $A_{\text {cond }}=62 \pm 2 \AA^{2}$ is consistent with the trident conformation of the SSS molecules in a monolayer film at the air-water interface. The cross-sectional area per hydrocarbon chain for SSS at $20^{\circ} \mathrm{C}$ in the $\alpha$-phase ( $\alpha$-phase has the most mobile acyl chains) is $19.7 \AA^{2}$ [13]. Our isotherms are consistent with earlier reports $[3,12]$.

\subsection{Isobaric compression}

Even though we found that the forced compression isotherms did not change appreciably for barrier velocities between 0.5 and $2 \mathrm{~cm} / \mathrm{min}$, under isobaric conditions, we did observe further compression though at velocities that were one or two orders of magnitude smaller. We stopped the forced compression when a certain surface pressure $\pi$ was reached. Next, we kept the surface pressure constant at that value, allowing the barrier to move. This is shown in Fig. 2.

After several minutes, a constant velocity was reached usually. The evolution of the trough length was fitted to

$l(t) \approx l_{0}-v\left(t-t_{0}\right)-\left(v_{\mathrm{f}}-v\right) h\left(t-t_{0}, a\right)$.

Here, the five fitting parameters are $l_{0}$, the trough length at the start of the isobaric compression, $t_{0}$, the starting time of the isobaric period, $v_{\mathrm{f}}$ and $v$, the forced and isobaric barrier velocity, respectively, and $a$, characterizing the transition from the forced to the isobaric regime. The

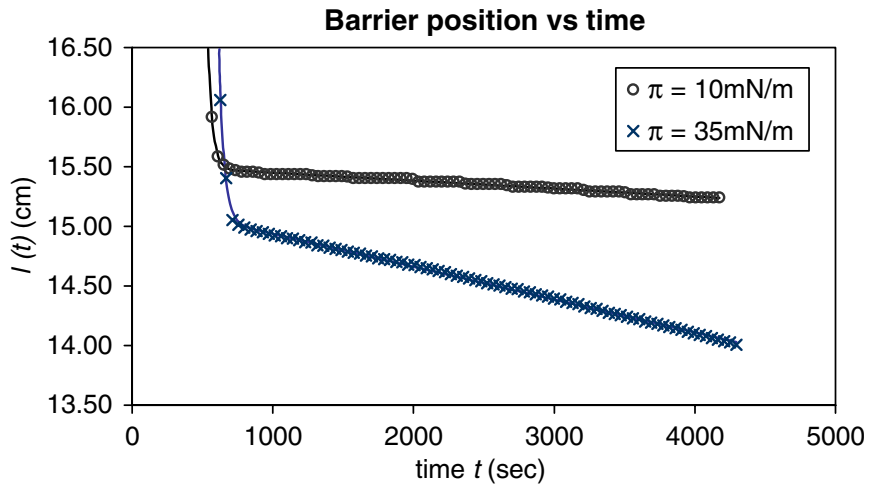

Fig. 2. Two examples of the measured barrier position as a function of time during forced and isobaric compression. The almost vertical parts of the curves correspond to forced compression at a rate of $1 \mathrm{~cm} / \mathrm{min}$. The slowly decreasing parts correspond to isobaric compression at the spreading pressure given in the figure. 
accuracy of the fits typically was $0.2 \%$. In all cases, the fitted forced velocity $v_{\mathrm{f}}$ was very close to the applied barrier velocity.

In Fig. 3, we show the dependence of the isobaric velocity $v$ on the surface pressure $\pi$. It can be noted that $v \approx 0$ for $\pi \leqslant 5 \mathrm{mN} / \mathrm{m}$ and depends linearly on $\pi$ for $5 \mathrm{mN} / \mathrm{m} \leqslant \pi \leqslant 35 \mathrm{mN} / \mathrm{m}$. For pressures $\pi \geqslant 42 \mathrm{mN} / \mathrm{m}$, i.e. beyond the collapse pressure, a much faster compression is found. These results show that the isotherm shown in Fig. 1 can be considered as an equilibrium isotherm only for $\pi \leqslant 5 \mathrm{mN} / \mathrm{m}$. For larger pressures, the equilibrium value of $A$ is smaller than the value displayed in Fig. 1. At this point, it is worthwhile to clearly discriminate between collapse pressure $\pi_{\mathrm{col}}$ and equilibrium pressure $\pi_{\mathrm{eq}}$. We use the definition of Roberts [14] in his book, whereas sometimes in the literature one means by equilibrium spreading pressure what we call collapse pressure, see e.g. Ref. [15]. Equilibrium (spreading) pressure is the surface pressure that is spontaneously generated when a crystalline sample of the solid material is placed in contact with the water surface. Provided that sufficient time is allowed for equilibration to occur, one can, in principle, be sure that the monolayer which has been formed by molecules detaching themselves from the crystal surface and spreading over the subphase is in equilibrium with the crystals themselves. At any surface pressure higher than this, there should be a tendency for the monolayer to aggregate into crystals [14]. According to our results (Fig. 3), equilibrium pressure for SSS at air-water interface is $\pi_{\mathrm{eq}}=5 \mathrm{mN} / \mathrm{m}$.

During the isobaric compression, some rearrangement must take place which effectively thickens the film. We assume that this process involves the growth of $3 \mathrm{D}$ crystals of SSS, and we investigate this hypothesis using AFM imaging. To this end, we compare LB films obtained by transfer at $t=t_{0}$ with films transferred 30 or $60 \mathrm{~min}$ after $t_{0}$.

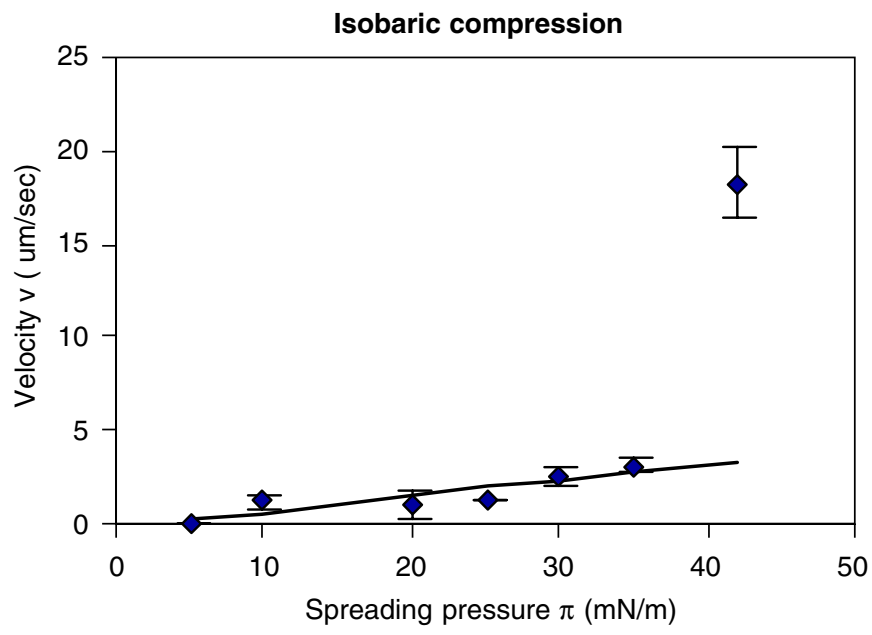

Fig. 3. Isobaric velocity $v(\mu \mathrm{m} / \mathrm{s})$ as a function of spreading pressure $\pi$, as obtained by fitting the measured barrier position to Eq. (3). Note the sharp increase of $v$ for spreading pressure above the collapse pressure $\pi_{\mathrm{col}}$.

\section{AFM observations}

\subsection{Monolayer thickness}

From the AFM images of LB films, withdrawn at $\pi=$ $5 \mathrm{mN} / \mathrm{m}$ (data not shown), it is seen that the mica is covered with a homogeneous monolayer. The monolayer can be successfully transferred to a mica surface and it is quite stable in the course of time. When the Langmuir film was prepared at higher pressures, a monolayer was observed as well, but now with embedded higher domains. After 1 day storage at room temperature of the withdrawn LB film, the monolayer is still present, though with slightly higher thickness (Fig. 4C, F).

We estimated the monolayer thickness $d_{0}=d_{0}(\pi)$ using the following procedure. We first scratched a rectangular hole in the monolayer with the AFM tip by scanning with a relatively large force $F \approx 30 \mathrm{nN}$. Then a larger image, including the hole was scanned with small forces $F=$ $1-8 \mathrm{nN}$ (Fig. 4). The height difference between the hole, and the surrounding gives an apparent thickness $d^{\prime}$. The fact that $d^{\prime}$ turned out to depend on the scanning force $F$ shows that the real monolayer thickness $d_{0}(\pi)$ depends on $d^{\prime}$.

In Fig. 5, we show data, together with an overall fit of the form

$d^{\prime}(\pi, F) \approx a+b \pi+c F+d \pi F$.

From this fit, we can estimate the real thickness $d_{0}(\pi) \approx d^{\prime}(\pi, F=0)$, corresponding to scanning force $F=0$, which is presented in Fig. 5 .

Note that the monolayer thickness varies from about 1.6 to $1.8 \mathrm{~nm}$ over the pressure range that we study here (Fig. 6). We interpret this change in thickness as reflecting a change in the tilt angle $\tau$ between the alkane chains and the substrate surface. Such a change in the tilt angle of amphiphilic molecules on air-water interface due to compression was reported before [16,17].

To translate the thickness into a tilt angle, we need to estimate the effective chain length. A first estimation we get from crystal data on the hexagonal $\alpha$-phase $[18,19]$. In this phase the SSS molecules, in tuning fork conformation, are parallel to the $c$-axis. Then the interplanar distance $d\left(\begin{array}{ll}0 & 0\end{array}\right)$, which is often referred to as long spacing, is equal to the length of the SSS molecule in tuning fork conformation. This length is built up from two times the chain length plus the length of the glycerol group, plus a small contribution from the contact region between SSS layers. Since $d\left(\begin{array}{lll}0 & 0 & 1\end{array}\right)=5.06 \mathrm{~nm}$ in the hexagonal $\alpha$-phase, the alkane chain length must be about $2.5 \mathrm{~nm}$. A more precise analysis and interpretation of crystallographic data of SSS in the

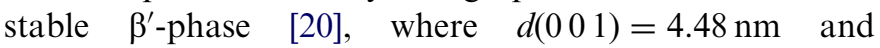
$\tau=60.8^{\circ}$, allows us to estimate an effective length of $5.13 \mathrm{~nm}$ of an SSS molecule in tuning fork conformation. Correcting this for the length of the glycerol and the contribution from the contact region in that phase, the alkane chain length can be estimated as $2.31 \mathrm{~nm}$. 
(A)
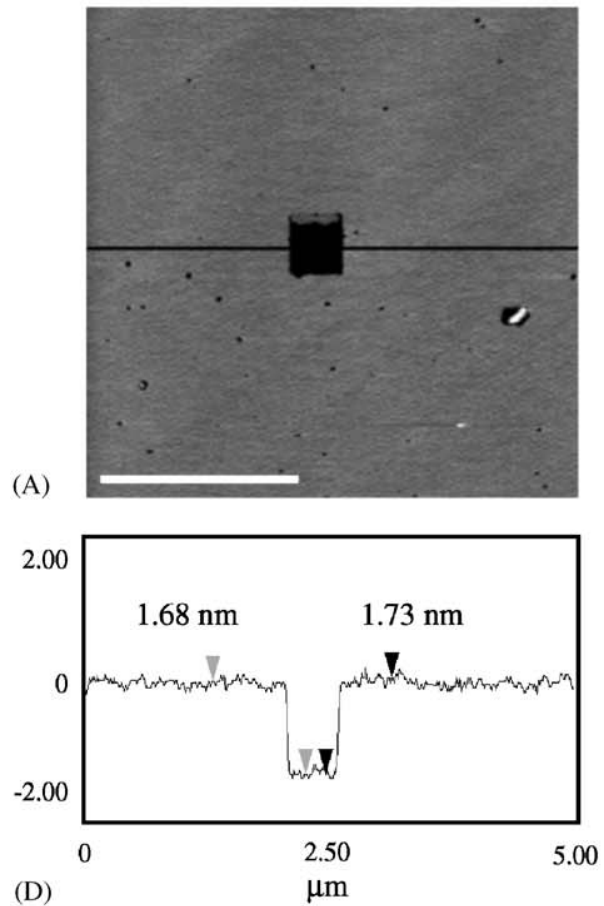

(B)
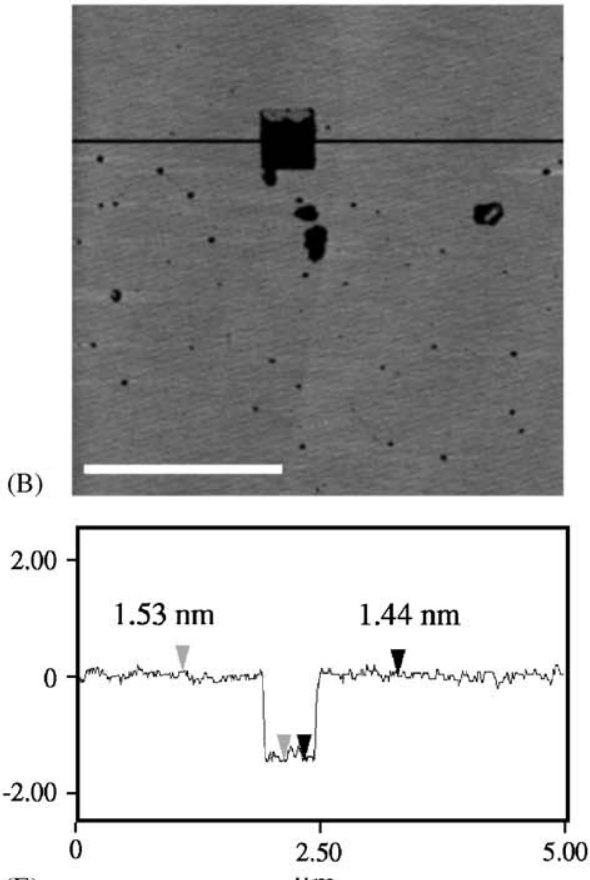

(E)
(C)
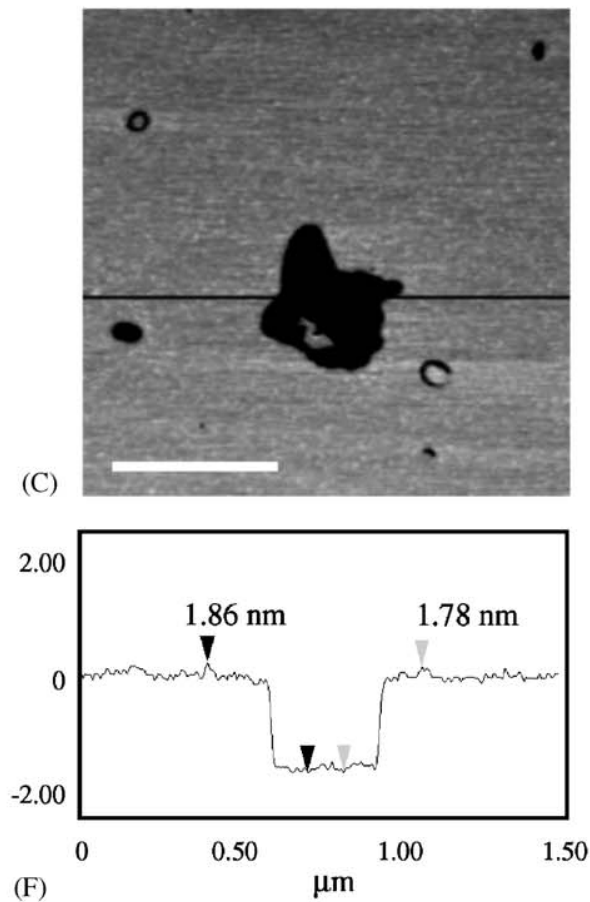

Fig. 4. AFM height image of an SSS monolayer transferred immediately after forced compression to surface pressure $\pi=30 \mathrm{mN} / \mathrm{m}$. The black squares are holes in the monolayer produced by scanning at a high force $(\sim 30 \mathrm{nN})$. (A) image scanned at AFM force $F=1 \mathrm{nN}$ with corresponding cross-section (D). (B) same area as in (A) scanned with AFM force $F=7.6 \mathrm{nN}$ and the corresponding cross-section (E). (C, F) same sample exposed to air at room temperature for 1 day at $F=1 \mathrm{nN}$. The scale bar is $2 \mu \mathrm{m}(\mathrm{A}, \mathrm{B})$ and $500 \mathrm{~nm}(\mathrm{C})$ and the vertical scale is $5 \mathrm{~nm}$ for all images.
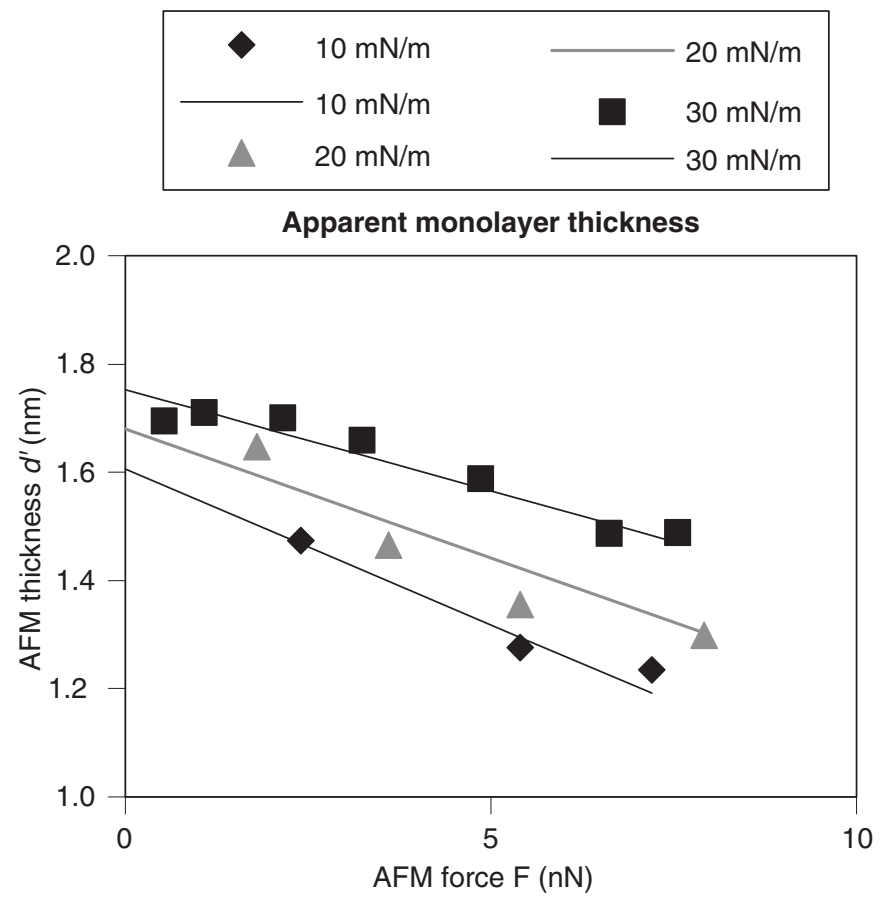

Fig. 5. Measured layer thickness $d^{\prime}$ as a function of applied AFM force $F$ and surface pressure $\pi$. The surface pressures $(\pi)$ at which the monolayer was compressed are given by the labels at the curves. The symbols correspond to the measured data and the lines are the fit according to Eq. (4).

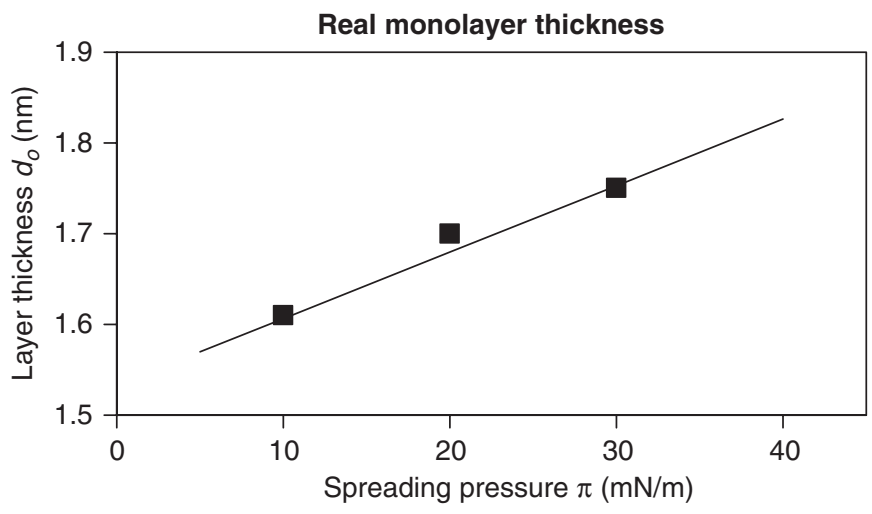

Fig. 6. Variation of the real thickness $d_{0}=d^{\prime}(F=0)$ of the monolayer with varying spreading pressures. Line: from the combined fit with Eq. (4), squares: from independent linear fits of $d^{\prime}(\pi, F)$ at fixed $\pi$.

We have no detailed information on the molecular conformation of the triglyceride molecules in the monolayer. In order to estimate the tilt angle in the monolayer, we assume that the glycerol part of the molecule makes close contact with the (hydrophilic) substrate. The alkane chains are stretched similar as in the $\alpha-, \beta$ - and $\beta^{\prime}$-phases, though in different orientation with respect to the glycerol group. This leads to a structure where alkane chains of $2.31 \mathrm{~nm}$ extend from the substrate to the monolayer surface at a height $d_{0}$ above the substrate. Thus, in the monolayer, 
SSS molecules adopt a trident conformation; we get a simple relation:

$\sin (\tau)=d_{0} /(2.31 \mathrm{~nm})$.

Interpreting our monolayer thickness data with Eq. (5), we see that the tilt angle varies from $\tau=43^{\circ}$ at $\pi=$ $10 \mathrm{mN} / \mathrm{m}$ to $\tau=53^{\circ}$ at $\pi=40 \mathrm{mN} / \mathrm{m}$. It is known that in the crystalline $\beta^{\prime}$ - and $\beta$-phases of triglycerides, the chains adopt specific tilt angles, which are characteristic for the chain-packing in the given triglyceride. In these phases, tilt angles always are above about $50^{\circ}$. Smaller tilt angles are energetically unfavourable $[1,19]$. Since presumably in the trident monolayer the alkane chains are less densely packed than the crystalline phases, a smaller tilt angle seems acceptable.

\subsection{Initial structure, obtained by forced compression}

Fig. 7 shows AFM images of SSS layers that we transferred from the water-air surface to mica, immediately after the spreading pressure $\pi$ was reached by forced compression. Domains are found that extend $3.5 \mathrm{~nm}$ or more above the monolayer level. Their density increases with increasing $\pi$ as shown in Fig. 8. We suggest that they are small initial crystals, formed in the period where the spreading pressure increases from the small values at which the film is in a two-dimensional gas state, to the final pressure $\pi$ at which the condensed phase has formed. In

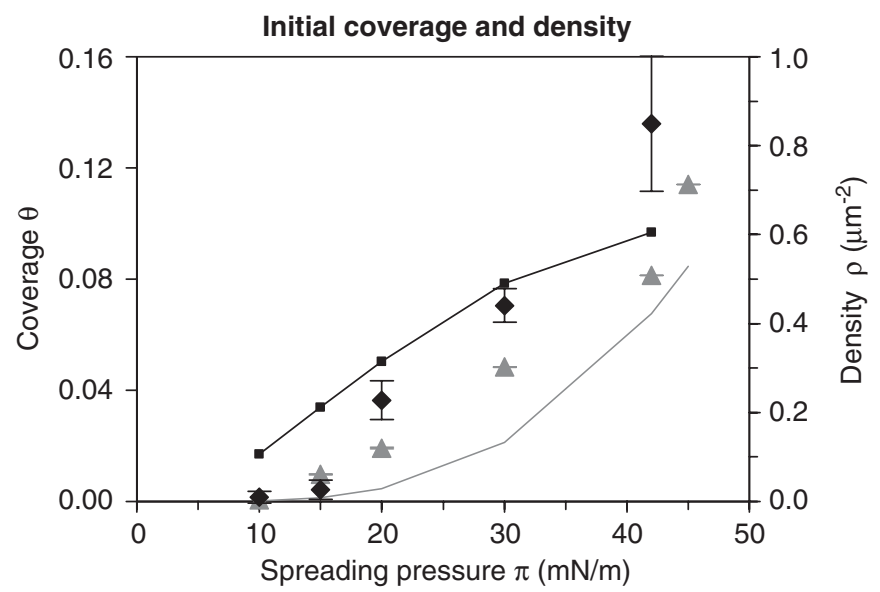

Fig. 8. Fraction $\theta$ of the film area, covered with crystals $(\boldsymbol{\Lambda})$, formed during the forced compression to spreading pressure $(\pi)$ and crystal density $\rho(\diamond)$. The curves are results obtained fitting all forced and isobaric compression image data to the model described in Section 6.
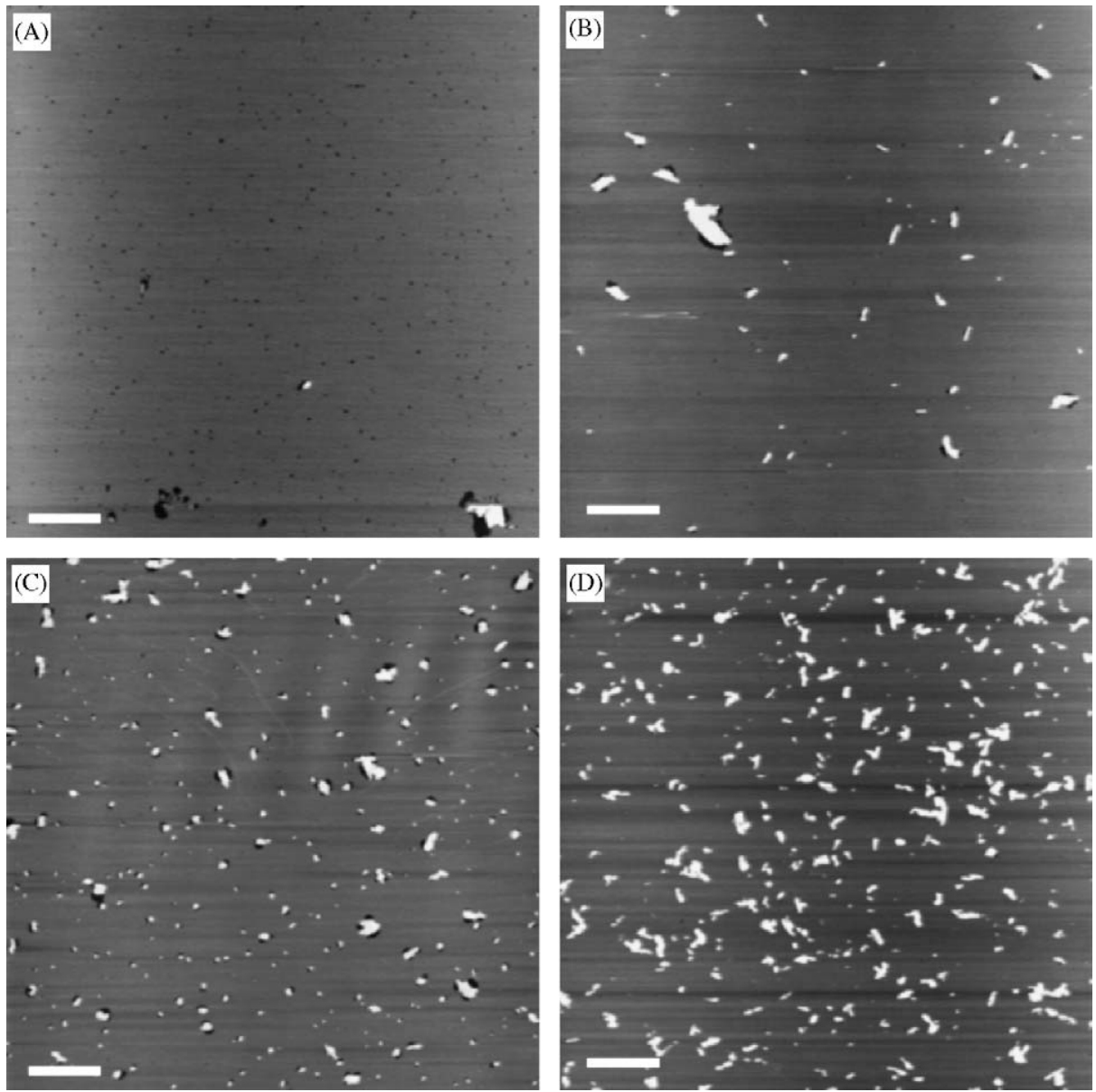

Fig. 7. AFM height image showing monolayers of SSS transferred immediately after forced compression to surface pressure (A) $\pi=10 \mathrm{mN} / \mathrm{m}$, (B) $\pi=20 \mathrm{mN} / \mathrm{m}$, (C) $\pi=30 \mathrm{mN} / \mathrm{m}$ and (D) $\pi=42 \mathrm{mN} / \mathrm{m}$. The density of higher domains, embedded in the monolayer, increases with the surface pressure. The scale bar is $2 \mu \mathrm{m}$ and the vertical scale is $20 \mathrm{~nm}$ for all images. 
this period, SSS molecules undergo major orientation and packing changes. Since the molecular surface density of the adsorbed film is already high, in the last part of this period, such motions are hindered considerably. As a result, the formation process of the domains will not be strictly deterministic and a metastable film structure may form. We suppose that the domains serve as crystal nuclei from which bigger crystals can grow when the Langmuir film is further compressed isobarically at constant pressure $\pi$.

\subsection{Structural changes during isobaric compression}

To investigate the structural changes of the Langmuir film in time, we transferred the Langmuir film to the mica surface 0,30 and $60 \mathrm{~min}$ after isobaric compression started. At surface pressure $\pi=5 \mathrm{mN} / \mathrm{m}$, we observed no significant differences between the monolayers withdrawn 0 or $30 \mathrm{~min}$ after the start of isobaric compression.

At a surface pressure $\pi=10 \mathrm{mN} / \mathrm{m}$, the AFM images show a homogeneous monolayer with small defects when the LB film was transferred to mica immediately after forced compression, as shown in Fig. 9A and D. After $30 \mathrm{~min}$ isobaric compression, we observed a few higher domains, embedded in the monolayer (Fig. 9B, E). These domains were soft and could be scratched away with the AFM tip, even at the normal scanning forces $F$ that are normally used for imaging. After several scans with $F=1-2 \mathrm{nN}$, the second layer disappeared, leaving a flat film with the same thickness as the trident monolayer,
Fig. 9C and F. The thickness of the domains, measured from the monolayer, was $3.5-3.6 \mathrm{~nm}$.

At surface pressure $\pi=20 \mathrm{mN} / \mathrm{m}$, we found that the directly transferred LB film consisted of an almost defectfree trident monolayer, in which many small domains were embedded. The thickness of the domains was found to be $4.8-5.1 \mathrm{~nm}$, as shown in Figs. 10A and D. On LB films transferred after $30 \mathrm{~min}$ isobaric compression, the domains within the trident monolayer were higher and bigger. The maximum measured thickness from the monolayer was $8.2 \pm 0.2 \mathrm{~nm}$ (Figs. 10B and E). After $60 \mathrm{~min}$ incubation, even higher domains were found with thickness up to $20 \pm 0.2 \mathrm{~nm}$ measured from the mica (Fig. 10C, F). On the highest domains, we found terraces separated by steps of height $4.9 \pm 0.1 \mathrm{~nm}$. In all cases, the domains were surrounded by the trident monolayer.

The same growth process was observed for LB layers obtained at surface pressure $\pi=30 \mathrm{mN} / \mathrm{m}$. A closer AFM observation showed us that on the bigger crystals formed at $\pi \geqslant 20 \mathrm{mN} / \mathrm{m}$, two different terraces can be found, with height thicknesses 3.5 and $5.1 \mathrm{~nm}$ from the monolayer (Fig. 11).

\subsection{Stability of the transferred LB film}

To check the stability of SSS layer in air, we transferred it immediately after forced compression to $\pi=30 \mathrm{mN} / \mathrm{m}$ and left it for 1 day at room temperature (Fig. 12). The monolayer became grainy and slightly higher. The crystals
(A)
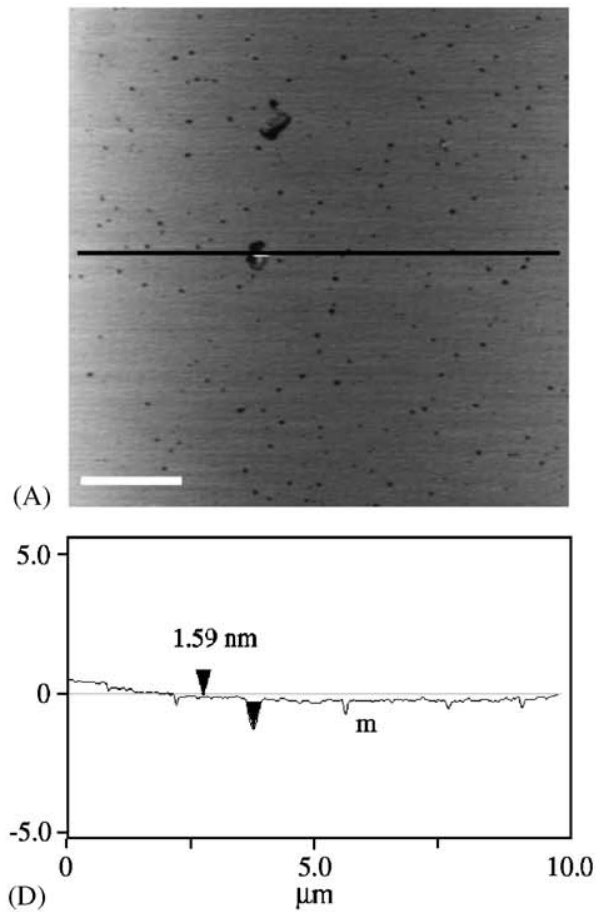

(B)
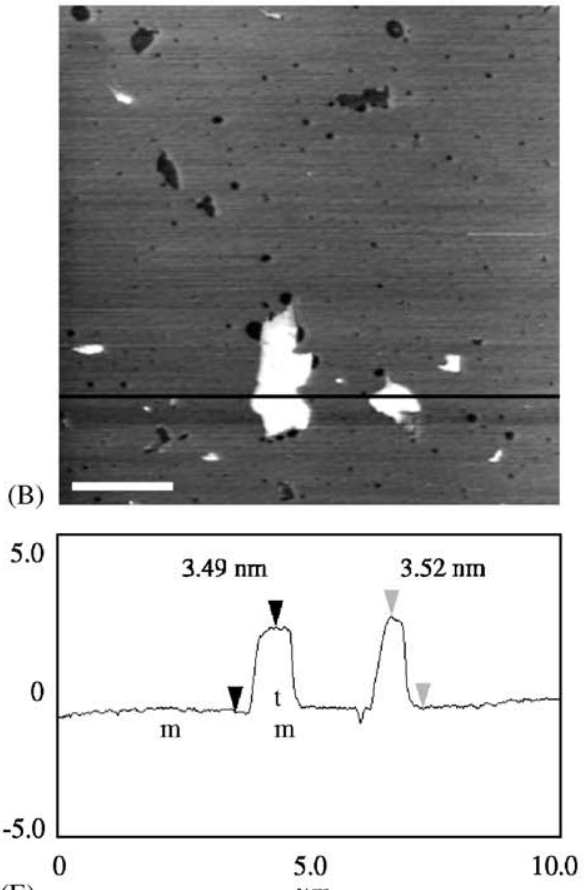

(C)
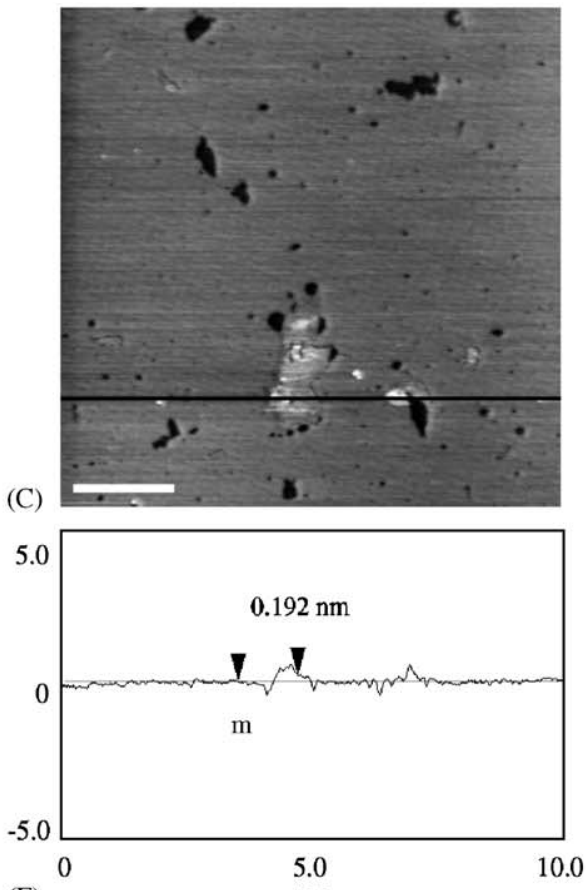

(E)

(F)

$\mu \mathrm{m}$

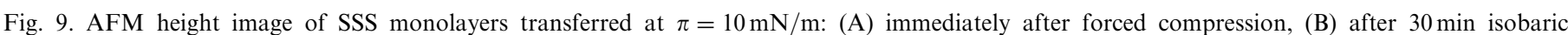

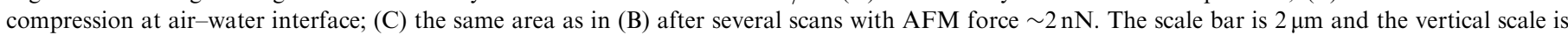

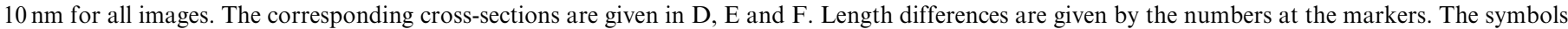
below the lines give our proposed structure of the crystals ( $m$ - trident conformation; $t$ - top layer tuning fork conformation). 

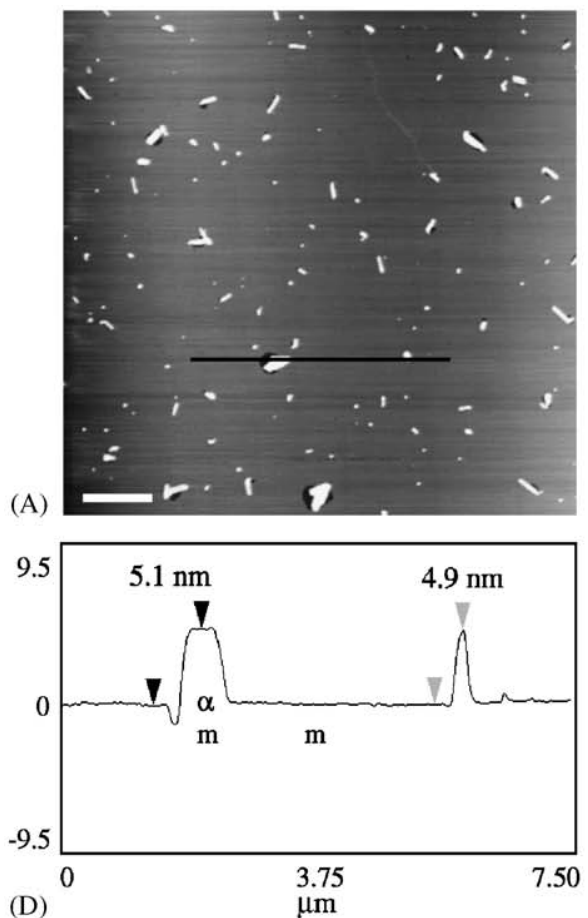

(B)
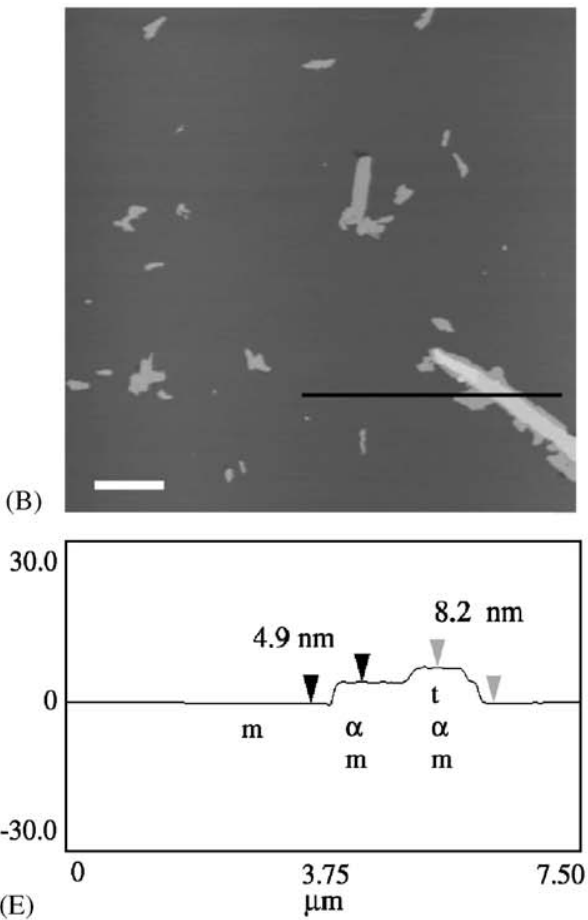

(E)

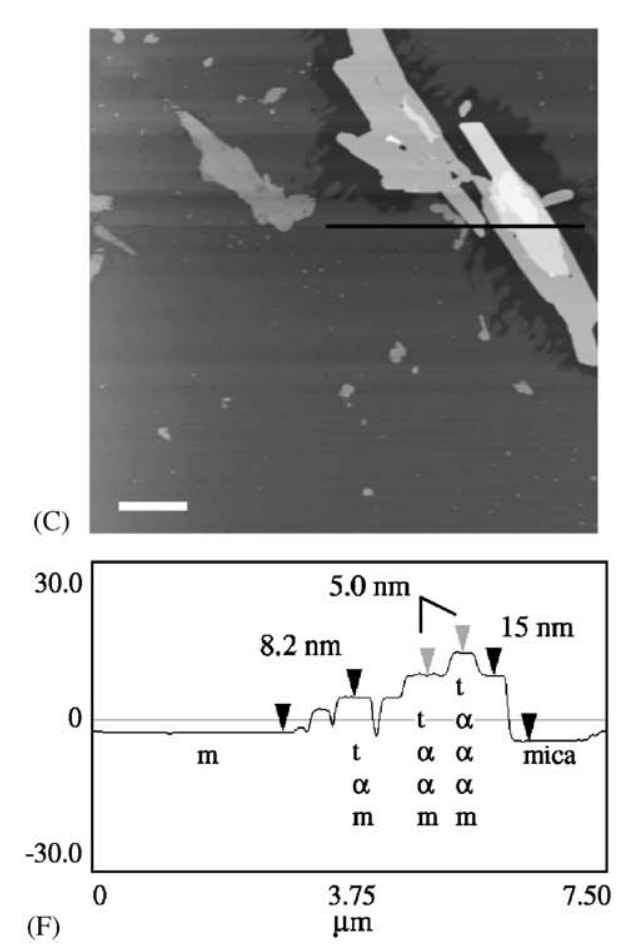

Fig. 10. AFM height image of SSS monolayers transferred at $\pi=20 \mathrm{mN} / \mathrm{m}$. (A) immediately after forced compression, (B) after $30 \mathrm{~min}$ isobaric compression at air-water interface and (C) after $60 \mathrm{~min}$ isobaric compression. The corresponding cross-sections are given in D, E and F. The scale bar is $2 \mu \mathrm{m}$ for all images and the vertical scale is $20 \mathrm{~nm}$ for (A) and $70 \mathrm{~nm}$ for (B, C). Length differences are given by the numbers at the markers. The symbols below the lines give our proposed structure of the crystals $(m$-trident conformation; $\alpha-$ crystal tuning fork conformation; $t$-top layer tuning fork conformation).

grew slightly and the newly grown parts of the crystals were $3.5 \pm 0.1 \mathrm{~nm}$ above the monolayer level. In some crystals, higher domains $(5.0 \pm 0.1 \mathrm{~nm})$ were observed. During the incubation in air of the transferred LB film, not only the already present crystals were growing, but also new very small nuclei appeared. We suggest that the grainy character of the monolayer is due to molecules, which leave the monolayer to form the new nuclei and the new parts of the present crystals.

\subsection{Consistency of Langmuir and AFM data}

If the densities of the monolayer and the higher domains were exactly the same, then the total film volume $V(t)$ should remain constant during the isobaric compression process (SSS is not volatile). In the Langmuir system, we measure the temporal change of the film area $A(t)$. From the AFM images, we can estimate the average film thickness $d(t)$. Ideally $A(t) d(t)=V$ is constant, whence $A(0) / A(t)$, which can be obtained from the Langmuir experiment, should be equal to $d(t) / d(0)$ as measured by AFM.

Fig. 13 shows that within the experimental accuracy, this is true. The relatively large uncertainty of $5-10 \%$ of $d(t)$ as estimated from AFM is due to the inherent inaccuracy of the standard Nanoscope "bearing analysis" software for estimating crystal volumes. The uncertainty in the Langmuir estimation of $2-4 \%$ is caused by the differences in the observed isobaric velocities $v$ as obtained from the fits in Section 3.2. As the individual fits are accurate, these velocity differences reflect accidental differences in the structure of the film that was being compressed.

\section{Theory for nucleation, growth and coalescence of crystals}

\subsection{Qualitative interpretation of film evolution observations}

In the sequel, we shall interpret the observed film structure on the basis of a model that is schematically presented in Fig. 14.

Our observations suggest that an SSS trident monolayer is thermodynamically unstable for spreading pressure $\pi \gtrsim 5 \mathrm{mN} / \mathrm{m}$. Therefore, during isobaric compression at $\pi \gtrsim 5 \mathrm{mN} / \mathrm{m}$, some SSS molecules move to the top of the monolayer. These molecules rearrange in higher domains where they presumably adopt the more stable tuning fork conformation and pack similar as in the crystalline $\alpha$ and $\beta$ crystal forms. This film structure was first proposed by Bursh and Larsson [3,21] to interpret the triple chain LBfilm thickness that they observed for LB films that were compressed beyond the collapse pressure. Based on a careful analysis of the observed domain height, we propose a new model for the structure and packing characteristics of the domains.

Using, as above, the estimated effective length of $5.13 \mathrm{~nm}$ for an SSS molecule in the tuning fork conformation, the 

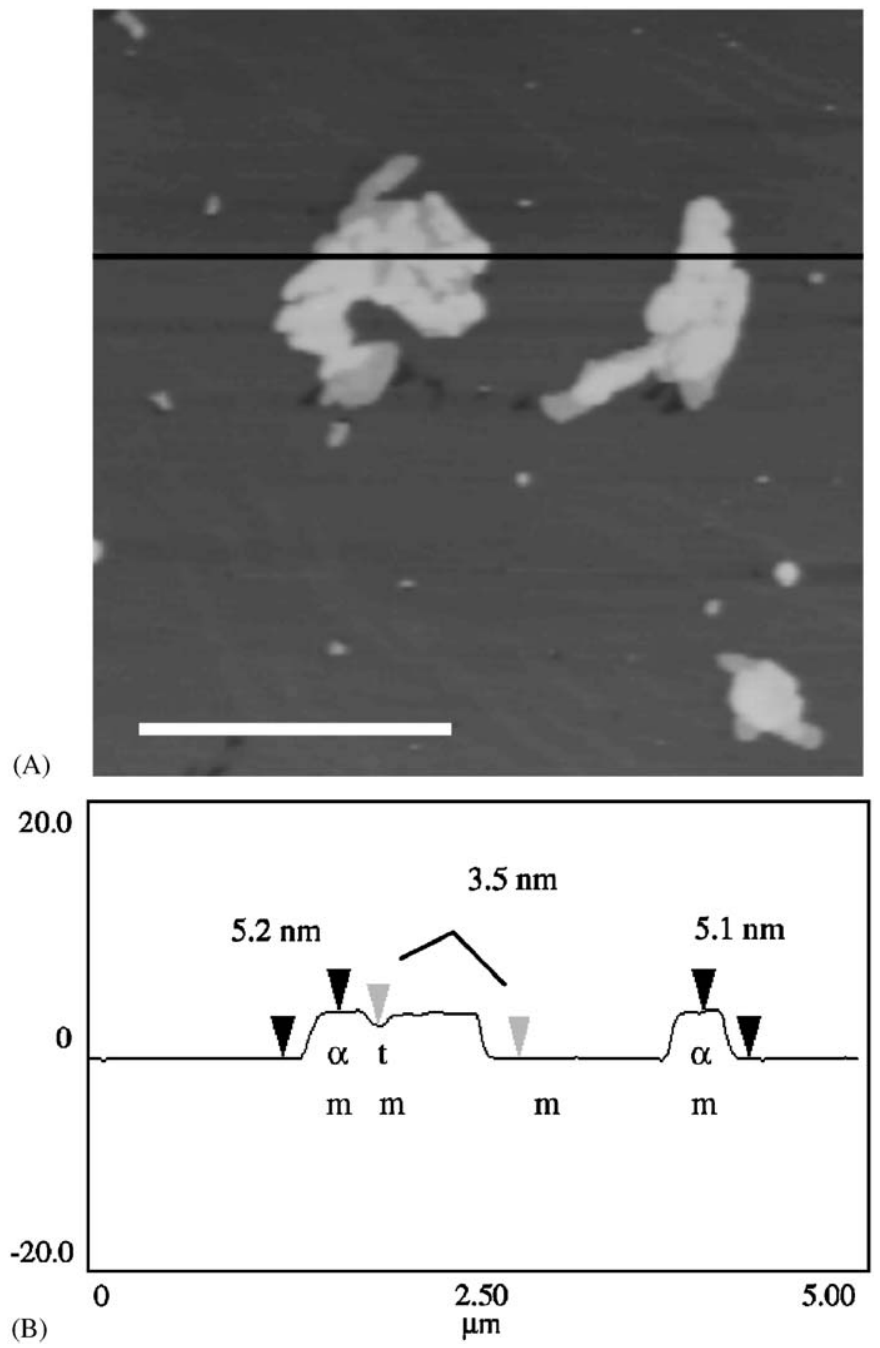

Fig. 11. AFM height image of SSS monolayer transferred at $\pi=$ $30 \mathrm{mN} / \mathrm{m}$ after $30 \mathrm{~min}$ isobaric compression at air-water interface (A). The corresponding cross-section is given in (B). The scale bar is $2 \mu \mathrm{m}$ and the vertical scale is $50 \mathrm{~nm}$.

observed domain thickness of $3.5 \mathrm{~nm}$ at $\pi=10 \mathrm{mN} / \mathrm{m}$ corresponds to a tilt angle $\tau=43-44.5^{\circ}$, i.e. somewhere between the estimated tilt angle in the trident monolayer and the tilt angle in the stable $\beta^{\prime}$-phase, Fig. 9B, E. We may suppose that the structure of these layers can be described as a slightly deformed $\beta$ - or $\beta^{\prime}$-phase. As we observed this layer thickness always at the upper film layer, we refer to this structure as the top layer structure (' $t$ ' in the figures). The fact that we have observed this structure to be common for top layers shows that the increased tilt is a form of surface relaxation, caused by the different interaction with other crystals layers.

At surface pressures $\pi \geqslant 20 \mathrm{mN} / \mathrm{m}$, some domains extended as much as $5.0 \pm 0.1 \mathrm{~nm}$ above the surrounding monolayer. This suggests that in these domains, the molecules are fully stretched $(5.13 \mathrm{~nm})$ and oriented perpendicular to the monolayer, i.e. the structure of these domains is similar to the crystalline $\alpha$-phase (Fig. 14C).
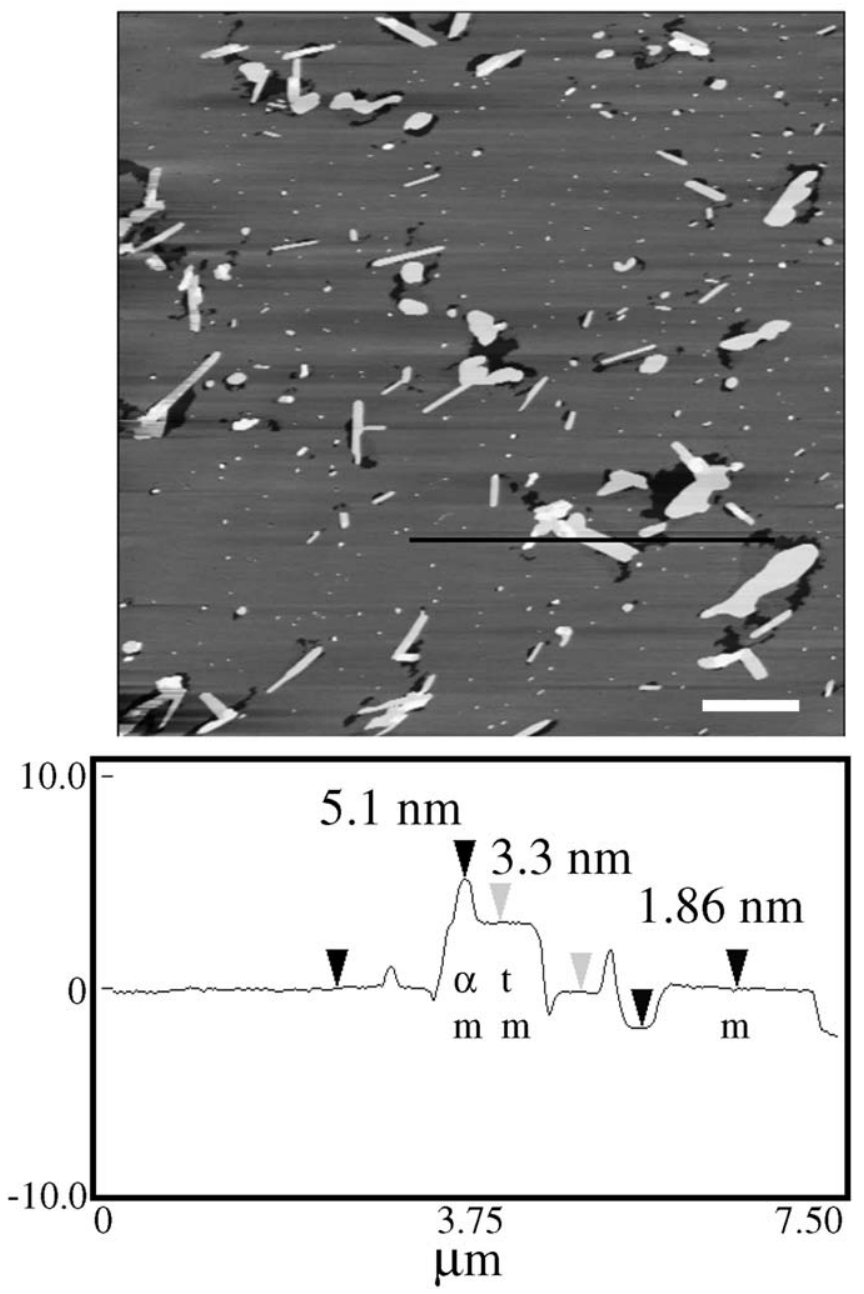

Fig. 12. AFM height image of a monolayer of SSS that was transferred to mica immediately after forced compression to surface pressure $\pi=$ $30 \mathrm{mN} / \mathrm{m}$ and that was left for 1 day in air at room temperature. The cross-section is shown in (B). The scale bar is $2 \mu \mathrm{m}$ and the vertical scale is $20 \mathrm{~nm}$.

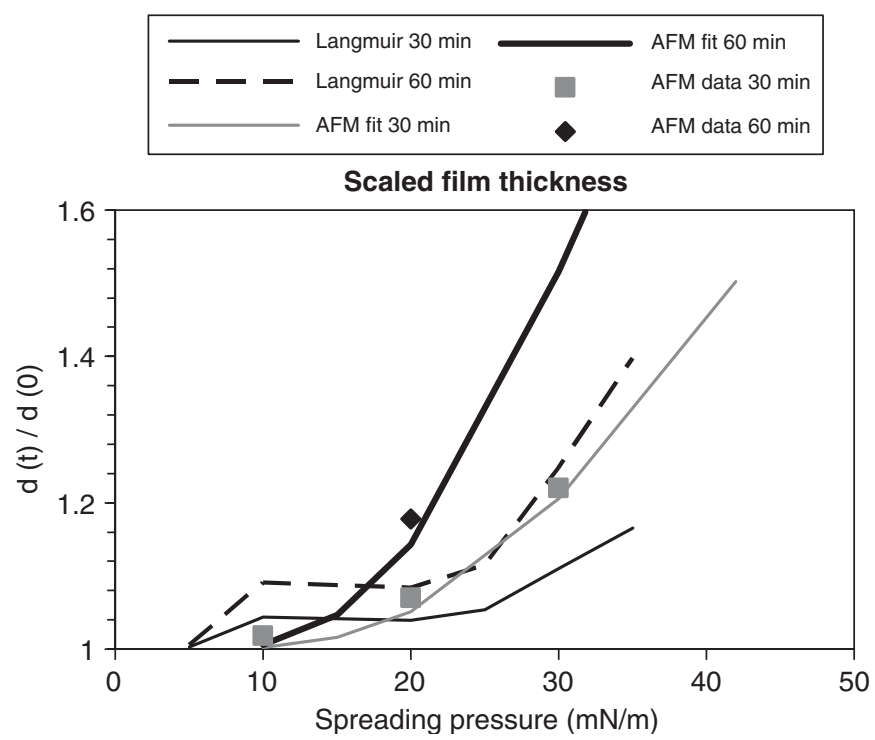

Fig. 13. Scaled film thickness estimated by Langmuir machine and AFM. 


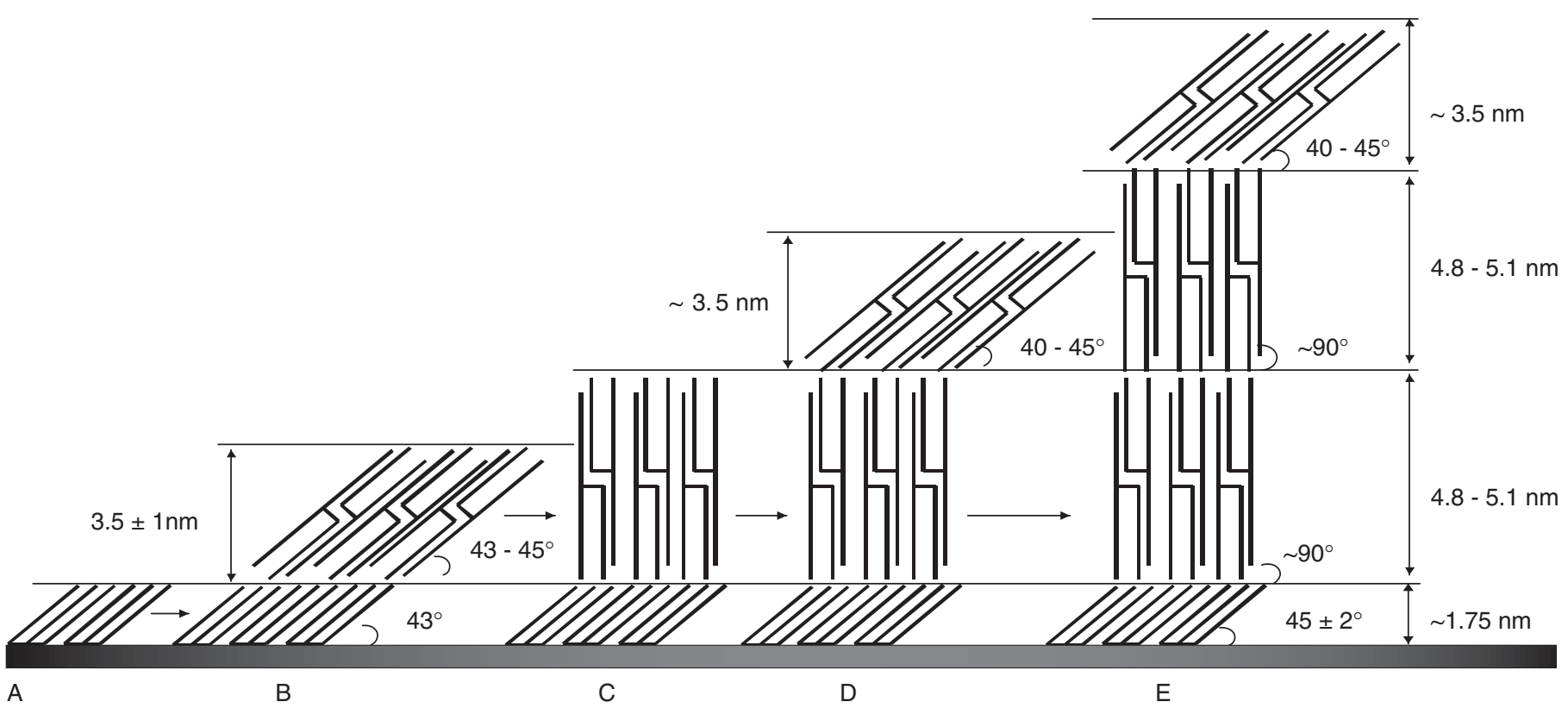

Fig. 14. Schematic illustration of the structures proposed for thin layers of SSS molecules. (A) Monolayer structure. At $\pi \leqslant 5 \mathrm{mN} / \mathrm{m}$, this is the only structure found; at $\pi \geqslant 10 \mathrm{mN} / \mathrm{m}$, it is the structure around the higher domains, (B) Structure of stable thin crystals. At $\pi=10 \mathrm{mN} / \mathrm{m}$, all observed crystals have this structure. (C) Structure of metastable crystals. Such crystals are found on films that are withdrawn immediately after forced compression to $\pi=20 \mathrm{mN} / \mathrm{m}$. (D, E) Structure of higher crystals. Such crystals are observed after 30 or $60 \mathrm{~min}$ isobaric compression at $\pi \geqslant 20 \mathrm{mN} / \mathrm{m}$.

During forced compression of the Langmuir film at $\pi \geqslant 20 \mathrm{mN} / \mathrm{m}$, the crystal growth process is so fast that this metastable, $\alpha$-like, polymorph with layer thicknesses

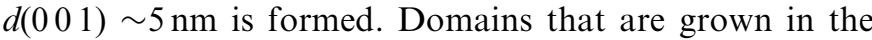
$\alpha$-phase will not spontaneously transform to the $\beta$ - or $\beta^{\prime}$-phase because this involves a very slow solid-solid transformation process.

For the slow growth process at $\pi=10 \mathrm{mN} / \mathrm{m}$, the stable $\beta$-phase is grown immediately, though with a top layer slightly thinner than the interplanar distance $d\left(\begin{array}{lll}0 & 0 & 1\end{array}\right)=$ $4.48 \mathrm{~nm}$ of the real $\beta^{\prime}$-phase (Fig. 9).

The higher domains $(8.2 \pm 0.2 \mathrm{~nm})$ found after isobaric compression can be explained with the formation of a second layer on top of the first one (Fig. 14D). This measured thickness does not correspond to two fully stretched layers $(\sim 10 \mathrm{~nm})$. We assume that the first layer is in $\alpha$-phase $(5.1 \mathrm{~nm})$ and the second layer, which in this case is the top layer, has the ' $t$ ' structure, having a tilt of $40-45^{\circ}$ and a thickness of $3.4 \pm 0.2 \mathrm{~nm}$. The reproducibility of the step height in the next layers, which is $4.8-5.1 \mathrm{~nm}$, supports this interpretation.

Combining our observations, we concluded that for $\pi \gtrsim 5 \mathrm{mN} / \mathrm{m}$, crystals of SSS in tuning fork conformation are growing on top of a trident monolayer at the air-water interface. If the growth is slow enough (e.g. at $\pi=$ $10 \mathrm{mN} / \mathrm{m}$ or in the last stages of growth at $\pi>$ $15 \mathrm{mN} / \mathrm{m}$ ), the crystals grow in the $\beta$ - or $\beta^{\prime}$-phase. The top layer is tilted at $43-45^{\circ}$, i.e. the molecules are somewhat more flat than in the $\beta$ - or $\beta^{\prime}$-phase. At larger growth rates (e.g. the initial stages of growth at $\pi>15 \mathrm{mN} / \mathrm{m}$ ), crystals are formed with a metastable $\alpha$-like structure. The transformation of $\alpha$-like crystals to a $\beta$ or $\beta^{\prime}$-like crystal structure is too slow to be observed. Only the top layer may relax to an inclined molecular orientation. The same crystal growth processes occur at the mica-air interface of the transferred LB film as at the air-water interface of the isobarricaly compressed Langmuir film, though much slower.

The type of crystal growth process proposed here, where the structure of the first layer is very different from that of the subsequent layers, is known as the Stranski-Krastanov growth mode.

\subsection{Parameters and measurable variables}

At this point, we know that the size of the crystals increases with time, the growth being mainly in lateral directions, and that the growth rate increases with surface pressure. The number of crystals too increases initially with time and with increasing surface pressure. In later stages, when a significant fraction of the monolayer film is covered by crystals, crystals start to coalesce, and their number decreases again. To interpret these observations more quantitatively, we develop a simple model.

Our model provides us with the dependence on time and on surface pressure of the crystal density $\rho\left(\mu \mathrm{m}^{-2}\right)$ the average crystal area $a\left(\mu \mathrm{m}^{-2}\right)$ and the fraction $\theta$ (dimensionless) of the film that is covered by crystals (area of the crystals divided by the total area of the image). At $t=0$, random nucleation of crystals starts at a rate $I$. We assume that the crystals have a roughly cylindrical form, with initial radius $R_{0}$ and height $h_{0}$, and that they grow with rates $v_{1}$ and $v_{v}$ in the lateral and vertical direction, respectively. We consider $I, R_{0}, h_{0}, v_{1}$ and $v_{v}$ as 
time-independent physical parameters that may depend on the spreading pressure of the film. Inspection of the data suggests that some nucleation of crystals takes place before the sample is removed from the Langmuir through. This means that a non-zero initial substrate coverage $\theta(t=0)$ and crystal density $\rho(t=0)$ have to be considered.

\subsection{Avrami-Kolmogorov theory for coverage}

In the beginning of the nucleation and growth process, the crystals are far enough away from each other to grow independently. We shall refer to the crystal density and the covered fraction in the initial stages as "free" values $\rho_{\mathrm{f}}$ and $\theta_{\mathrm{f}}$ :

$$
\begin{aligned}
\rho_{\mathrm{f}}(t) & =\rho_{\mathrm{f}}(0)+\int_{0}^{t} I\left(t^{\prime}\right) \mathrm{d} t^{\prime}=\rho_{\mathrm{f}}(0)+I t, \\
\theta_{\mathrm{f}}(t) & \equiv \theta_{\mathrm{f} 0}(t)+\int_{0}^{t} I\left(t^{\prime}\right) \cdot \pi\left(R_{0}+v_{l}\left(t-t^{\prime}\right)\right)^{2} \mathrm{~d} t^{\prime} \\
& =\theta_{\mathrm{f} 0}(t)+\pi I\left(R_{0}^{2} t+v_{l} R_{0} t^{2}+\frac{1}{3} v_{l}^{2} t^{3}\right) .
\end{aligned}
$$

The first terms in Eqs. (6) and (7) describe the effect of crystals that were already present at the beginning, $t=0$, of the isobaric compression. Their density does not change in time, but their coverage grows according to

$\theta_{\mathrm{f} 0}(t)=\rho_{\mathrm{f}}(0) \pi\left(\sqrt{\frac{\theta_{\mathrm{f}}(0)}{\pi \rho_{\mathrm{f}}(0)}}+v_{l} t\right)^{2}$.

This expression can be found by using that the average area $\pi R(t=0)^{2}$ of the crystals at $t=0$ equals $\vartheta_{\mathrm{f}}(0) / \rho_{\mathrm{f}}(0)$.

In the later stages of film growth, the actual values of $\rho(t)$ and $\theta(t)$ will be smaller than the free values, $\rho(t)<\rho_{\mathrm{f}}(t)$ and $\theta(t)<\theta_{\mathrm{f}}(t)$. The fact that crystals can only grow and nucleate in the uncovered film area between the already existing crystals is captured by the Avrami-Kolmogorov theory, leading to

$\theta(t)=1-\exp \left(-\theta_{\mathrm{f}}(t)\right)$.

This expression gives us the actual coverage of the film by crystals. It depends, however, on too many physically important parameters to hope that all these parameters can be derived from observed $\theta(t)$ curves alone. Therefore, we want to use observed crystal sizes and crystal densities as well. What we need is an expression for the number $N_{\mathrm{c}}=$ $N_{\mathrm{c}}(t)$ of free crystals that have merged to form one actual crystal. Then the experimental crystal density and crystal size are found from

$\rho=\rho_{\mathrm{f}} / N_{\mathrm{c}}$,

$a=\theta / \rho=\theta N_{\mathrm{c}} / \rho_{\mathrm{f}}$.

Unfortunately, no general theory to obtain $N_{\mathrm{c}}$ is available. In the next section, we develop an approach to the problem.

\subsection{Approximate theory for average crystal size and density}

In the spirit of the Avrami-Kolmogorov theory, the first step is to consider the growing film as if circular crystals nucleate and grow independently. For the free crystal coverage and density, we use Eqs. (6) and (7). The average area $a_{\mathrm{f}}$ of freely growing crystals is not simply the area $\pi\left(R_{0}+v_{l} t\right)^{2}$ of crystals that nucleate at time $t=0$. Crystals that nucleate later have a smaller area at time $t$. Taking this into account, we obtain

$$
\begin{aligned}
& a_{\mathrm{f}}(t)=\theta_{\mathrm{f}}(t) / \rho_{\mathrm{f}}(t)=\pi\left(R_{0}^{2}+R_{0} v_{l} t+\frac{1}{3} v_{l}^{2} t^{2}\right), \\
& R_{\mathrm{f}}(t) \equiv \sqrt{\frac{a_{\mathrm{f}}}{\pi}}=\sqrt{R_{0}^{2}+R_{0} v_{l} t+\frac{1}{3} v_{l}^{2} t^{2}},
\end{aligned}
$$

for the average area $a_{\mathrm{f}}$ and radius $R_{\mathrm{f}}$ of freely growing crystals.

In the next step, we take the merging and overlapping of these free crystals into account. Two crystals merge to form one new crystal if they are located close enough together. Two circular crystals with radius $R_{\mathrm{f}}$ touch each other, and will probably coalesce, if their (centre-to-centre) distance is $2 R_{\mathrm{f}}$ or less. Generally, we assume that two crystals merge if one is located within the merging region $A^{+}$of the other. The area of $A^{+}$is written as $a^{+}=\eta^{2} a_{\mathrm{f}}$, with $\eta \approx 2$.

The key idea is to define $\rho_{\mathrm{c}}(\mathbf{r})$ as the density of original crystals that are, directly or indirectly, connected to a original crystal in the origin. This density satisfies

$\rho_{\mathrm{c}}(\mathbf{r})=\rho_{\mathrm{f}}\left(1-P_{0}(r)\right)$.

Here, $P_{0}(\mathbf{r})$ is the probability that an original crystal at $\mathbf{r}$ is not connected to the crystal in the origin. Let $A^{+}(\mathbf{r})$ be the merging region of a crystal at $\mathbf{r}$. All original crystals in the merging region $A^{+}(0)$ of the central original crystal are connected to this crystal, hence $\rho_{\mathrm{c}}(\mathbf{r})=\rho_{\mathrm{f}}$ for $\mathbf{r}$ inside $A^{+}(0)$. Further away, the probability of a given crystal at $\mathbf{r}$ to be considered to the central crystal is equal to the probability to find at least one connected crystal in its merging region $A^{+}(\mathbf{r})$. Therefore, $\rho_{\mathrm{c}}(\mathbf{r})$ satisfies the implicit equation

$\rho_{\mathrm{c}}(r)=\left\{\begin{array}{cc}\rho_{\mathrm{f}} & \text { for } r \in A^{+}(0), \\ \rho_{\mathrm{f}}\left[1-\exp \left(-\int_{A^{+}(r)} \rho_{\mathrm{c}}\left(r^{\prime}\right) \mathrm{d}^{2} r^{\prime}\right)\right] & \text { for } r \notin A^{+}(0) .\end{array}\right.$

The latter expression derives from the $N=0$ case of the probability

$P_{N}=\frac{1}{N !}(\rho a)^{N} \exp (-\rho a)$

to find $N$ objects within an area $a$, when the average density of these objects is $\rho$.

Eq. (15) is an integral equation for $\rho_{\mathrm{c}}(\mathbf{r})$, which cannot be solved exactly. We make the following observations. If the free crystal density $\rho_{\mathrm{f}}$ is so high that the summed merging area is larger than the total film area, $a^{+} \rho_{\mathrm{f}}=\eta^{2} \theta_{\mathrm{f}}>1$, then 
far enough outside $A^{+}(0)$, a constant solution

$\rho_{\mathrm{c}}(\mathbf{r})=\rho_{\mathrm{c}}=\rho_{\mathrm{f}}\left(1-\exp \left(-a^{+} \rho_{\mathrm{c}}\right)\right)$

exists. In this case, merging of the original crystals leads to an infinite crystal.

For smaller free crystal densities $\rho_{\mathrm{f}}$, the connected crystal density $\rho_{\mathrm{c}}(\mathbf{r})$ decays to 0 with increasing distance from the central crystal. Assuming that $\rho_{\mathrm{c}}(\mathbf{r})$ varies slowly over the circular region $A^{+}(\mathbf{r})$ of radius $\eta R_{\mathrm{f}}$ and area $a^{+}=\pi \eta^{2} R_{\mathrm{f}}^{2}$, we have for the integrand in Eq. (15)

$\int_{A^{+}(r)} \rho_{\mathrm{c}}\left(r^{\prime}\right) d^{2} r^{\prime} \approx a^{+} \rho_{\mathrm{c}}(r)+\frac{1}{8}\left(a^{+}\right)^{2} \nabla^{2} \rho_{\mathrm{c}}(r)+\ldots \ldots$,

where $\nabla^{2}$ is the two-dimensional Laplace operator. Here, the first two terms of a Taylor expansion of $\rho_{\mathrm{c}}(\mathbf{r})$ have been used.

Substituting Eq. (18) in Eq. (15) for $\mathbf{r} \notin A^{+}(0)$, we get for $a^{+} \rho_{\mathrm{c}} \ll 1$ a linear differential equation

$\rho_{\mathrm{c}}(r)=\rho_{\mathrm{f}}\left(a^{+} \rho_{\mathrm{c}}(r)+\frac{1}{8}\left(a^{+}\right)^{2} \nabla^{2} \rho_{\mathrm{c}}(r)\right)$.

The general solution that vanishes at infinity is

$\rho_{\mathrm{c}}(r)=\rho_{\mathrm{c}}(r)=c \rho_{\mathrm{f}} \frac{K_{0}\left(\beta r /\left(\eta R_{\mathrm{f}}\right)\right)}{K_{0}(\beta)}$,

where $K_{0}$ is a Bessel function. The scaling factor $\beta$ can be expressed in terms of the free coverage $\theta_{\mathrm{f}}$ as given by Eqs. (7)-(12):

$\beta \equiv \sqrt{\frac{8\left(1-\eta^{2} \theta_{\mathrm{f}}\right)}{\pi \eta^{2} \theta_{\mathrm{f}}}}$.

The constant $c$ is obtained from a matching criterion at the edge of the merging region $A^{+}(0)$ of the central crystal, i.e. at $r=\eta R_{\mathrm{f}}$. Of the circular region $A^{+}(\mathbf{r})$ with the same area $a^{+}$at the edge of $A^{+}(0)$, a fraction $\gamma=2 / 3-\sqrt{3} /(2 \pi)$ overlaps with $A^{+}(0)$. In that part, all crystals are connected to the central crystal, whence $\rho_{\mathrm{c}}(\mathbf{r})=\rho_{\mathrm{f}}$. On the remaining fraction $1-\gamma$ of $A^{+}(\mathbf{r})$, we approximate the density of connected crystals by the density at the edge: $\rho_{\mathrm{c}}(\mathbf{r}) \approx \rho_{\mathrm{c}}\left(\eta R_{\mathrm{f}}\right)$. Thus, we approximate the surface integral in Eq. (15) by

$\int_{A^{+}(\mathbf{r})} \rho_{\mathrm{c}}\left(\mathbf{r}^{\prime}\right) d^{2} r^{\prime} \approx a^{+}\left[\gamma \rho_{\mathrm{f}}+(1-\gamma) \rho_{\mathrm{c}}\left(\eta R_{\mathrm{f}}\right)\right]$.

Substituting Eq. (20) with $r=\eta R_{\mathrm{f}}$ into Eq. (15) leads to an implicit equation for $c$ :

$c=1-\exp \left(-\eta^{2} \theta_{\mathrm{f}}[\gamma+(1-\gamma) c]\right)$,

from which $c$ can be solved by numerical iteration.

Upon integrating $\rho_{\mathrm{c}}(\mathbf{r})$ over the whole surface, we obtain the average number of original crystals that are, directly or indirectly, connected to a given cluster, i.e. for the average number $N_{\mathrm{c}}$ of original crystals that merge to form a new crystal:

$$
\begin{gathered}
N_{\mathrm{c}} \equiv 1+\int \rho_{\mathrm{c}}(r) d^{2} r \approx 1+2 \pi \int_{0}^{\eta R_{f}} \rho_{f} r d r+2 \pi \\
\int_{0}^{\eta R_{\mathrm{f}}} \rho_{\mathrm{c}}(r) r d r=1+\eta^{2} \theta_{\mathrm{f}}\left(1+\frac{\eta^{2} c}{\beta^{2}} \frac{K_{1}(\beta)}{K_{0}(\beta)}\right) .
\end{gathered}
$$

Note that this average crystal size diverges if the free coverage $\theta_{\mathrm{f}}$ approaches the limiting value $\eta^{2} \theta_{\mathrm{f}} \rightarrow 1$ where we found that Eq. (15) had an infinite cluster solution Eq. (17).

\subsection{Interpretation of AFM images of nucleation and growth}

Combining Eq. (24) with Eqs. (6)-(11), we can express the observed film coverage, crystal density and crystal size in terms of the nucleation rate and the lateral growth rate. To interpret data on the individual crystal volumes as well, we assume that the nuclei have a monolayer height and that vertical growth takes place on top of these with a constant velocity $v_{v}$. We have simultaneously fitted all our AFM data to Eqs. (6)-(11), (24). The results of the fit are given in the table below.

\begin{tabular}{llll}
\hline & $\begin{array}{l}\text { Symbol } \\
\text { in text }\end{array}$ & Value & Unit \\
\hline $\begin{array}{l}\text { Equilibrium } \\
\text { pressure }\end{array}$ & $\pi_{\mathrm{eq}}$ & $5.0 \pm 0.5$ & $\mathrm{mN} / \mathrm{m}$ \\
$\begin{array}{l}\text { Initial } \\
\text { density }\end{array}$ & $\rho_{\mathrm{f}}(t=0)$ & $\begin{array}{l}(0.020 \pm 0.002) \\
\left(\pi-\pi_{\mathrm{eq}}\right)\end{array}$ & $\mu \mathrm{m}^{-2}$ \\
$\begin{array}{l}\text { Initial } \\
\text { radius }\end{array}$ & $R_{0}$ & $(4.5 \pm 0.5)\left(\pi-\pi_{\mathrm{eq}}\right)$ & $\mathrm{nm}$ \\
$\begin{array}{l}\text { Nucleation } \\
\text { rate }\end{array}$ & $I$ & $<0.0002\left(\pi-\pi_{\mathrm{eq}}\right)$ & $\mu \mathrm{mm}^{-2}$ \\
$\begin{array}{l}\text { Lateral } \\
\text { growth rate }\end{array}$ & $v_{1}$ & $\begin{array}{l}(0.45 \pm 0.05) \\
\left(\pi-\pi_{\mathrm{eq}}\right)\end{array}$ & $\mathrm{nm} / \mathrm{min}$ \\
$\begin{array}{l}\text { Vertical } \\
\text { growth rate }\end{array}$ & $v_{v}$ & $\begin{array}{l}(0.0009 \pm 0.0001) \\
\left(\pi-\pi_{\mathrm{eq}}\right)\end{array}$ & $\mathrm{nm} / \mathrm{min}$ \\
\hline
\end{tabular}

The first fit parameter is the equilibrium value $\pi_{\mathrm{eq}}$ of the spreading pressure. Nucleation and growth of crystals is expected to take place in the film only for pressures $\pi-\pi_{\mathrm{eq}}$. For the other model parameters, we compared the quality of the fit for the two cases that the parameter was taken independent of $\pi$ or proportional to $\pi-\pi_{\text {eq }}$. In all cases, the second choice performed better. We also found that the fitting model did not produce a significantly positive value for the nucleation rate (after withdrawal of the film from the through). As a matter of fact, direct inspection of the AFM images confirmed that only rarely the number of crystal increased with increasing time $t$ after withdrawal. The decrease in the number of crystals due to coalescence and merging was a more dominant process. Therefore, we took $I=0$ to estimate the other model parameters (thus leaving us with a 5-parameter fit to 4 independent 
observables. We used 10 different combinations of time and spreading pressure (altogether 78 AFM images were taken into account). For the nucleation rate, we give an upper limit.

In view of the uncertainty and experimental spread of the AFM images, the fits are quiet satisfactory. Using more elaborate models does not seem justified or useful.

\section{Conclusions}

In this study, we have obtained AFM images that reveal the structure of thin tristearin (SSS) films, formed at air-water interface. Based on Langmuir and AFM experiments, we estimated crystal growth rates in the lateral and vertical direction. Our investigations lead to the following conclusions.

Compressing an extended SSS film at the air-water interface slowly, starting at very low surface pressure, monolayers of SSS molecules in trident conformation are formed. These monolayers are thermodynamically stable at surface pressure $\pi \leqslant 5 \mathrm{mN} / \mathrm{m}$. This is the equilibrium spreading pressure for this system. At higher surface pressures, a monolayer with a trident molecular conformation is still formed, but it is metastable and transforms slowly to form crystalline domains. The monolayer can be successfully transferred onto a mica surface and the resulting Langmuir-Blodgett film is quite stable in the course of time. Using AFM imaging, the monolayer thickness of the trident monolayer can be measured. We find that the thickness depends on the surface pressure, which we interpret as a change in the tilt angle between the average chain direction and the crystal surface. From thicknesses between 1.6 and $1.8 \mathrm{~nm}$, we conclude that the tilt angle gradually increases from $\pi=43^{\circ}$ at $\pi=10 \mathrm{mN} / \mathrm{m}$ to $\pi=53^{\circ}$ at $\pi=40 \mathrm{mN} / \mathrm{m}$.

At pressures $\pi \geqslant 10 \mathrm{mN} / \mathrm{m}$, the trident monolayer is thermodynamically metastable. Macroscopically it is immediately determined by the behaviour of Langmuir films during isobaric compression. The film area decreases slowly but steadily when the film is subject to a constant surface pressure. We investigated this process on the molecular scale by comparison of AFM images of LB films that were produced after different periods of isobaric compression. We concluded that crystal growth takes place under these conditions. Within the crystals, the molecules adopt a tuning fork conformation, except for the bottom layer where the molecules stay in the trident conformation. The crystal sizes increase with time, the growth being mainly in lateral directions. The growth rate increases with surface pressure.

We developed a new model to describe the growth and coalescence of crystals in the film. Fitting the AFM data to this model, we estimate a lateral growth rate of $2.3 \mathrm{~nm} / \mathrm{min}$ and a vertical growth rate of $0.005 \mathrm{~nm} / \mathrm{min}$ at $\pi=10 \mathrm{mN} / \mathrm{m}$. The data were insufficient to get a reliable estimate of the nucleation rate of new crystals, we merely estimate an upper limit of 0.001 new nuclei per $\mu \mathrm{m}^{2}$ and per minute.

At and above the collapse pressure, the crystal growth process is the same, though faster than in the intermediate regime. The isobaric compression velocity increases rapidly from $3 \mu \mathrm{m} / \mathrm{s}$ at $\pi=35 \mathrm{mN} / \mathrm{m}$ to $18 \mu \mathrm{m} / \mathrm{s}$ at $\pi=42 \mathrm{mN} / \mathrm{m}$.

The transformation processes that took place in the Langmuir film (floating on water) were observed in the transferred LB film (on mica) as well. In the latter case, however, the processes were orders of magnitude slower.

\section{Acknowledgements}

The authors would like to thank Marija Matovic for the fruitful discussions.

\section{References}

[1] N. Garti, K. Sato, in: Crystallization and Polymorphism of Fats and Fatty Acids, Dekker, M., New York, USA, 1988.

[2] M. Ollivon, Triglycerides, in: A. Karieskind (Ed.), Manuel des Corps Gras, Lavoisier, Paris, France, 1992, p. 469.

[3] T. Bursh, K. Larsson, M. Lundquist, Chem. Phys. Lipids 2 (1968) 102.

[4] J.A. Hamilton, D.M. Small, Proc. Nat. Acad. Sci. USA 78 (1981) 6878.

[5] J.A. Hamilton, Biochemistry 28 (1989) 2514.

[6] P.M. Claesson, A. Dedinaite, B. Bergenstahl, B. Campbell, H. Christenson, Langmuir 13 (1997) 1682.

[7] M. Lundquist, in: Surface Chemistry, Copenhagen. Munksgaard, 1966, p. 294.

[8] I. Kuzmenko, R. Buller, W.G. Bouwman, K. Kjaer, J. AlsNielsen, M. Lahav, L. Leiserowitz, Science 274 (1996) 20046.

[9] J.Z. Xue, C.S. Jung, M.W. Kim, Phys. Rev. Lett. 69 (1992) 474.

[10] P. Ekwall, R. Ekholm, A. Norman, Acta Chem. Scand. 11 (1957) 703.

[11] P.J. Birker, J.C. Blonk, J. Am. Oil Chem. Soc. 70 (1993) 319.

[12] M. Michalski, P. Brogueira, A. Goncalves da Silva, B. Saramago, Eur. J. Lipid Sci. Technol. 103 (2001) 677.

[13] C. Akita, T. Kawaguchi, F. Kaneko, O. Yamamuro, H. Akita, M. Ono, M. Suzuki, J. Crystal Growth 275 (2005) e2187.

[14] G. Roberts, Langmuir-Blodgett Films, Plenum Press, New York, 1990 , p. 21.

[15] G.L. Gaines, Insoluble Monolayers at Liquid-Gas Interface, Wiley, New York, 1966.

[16] S. Karaborni, S. Toxvaerd, J. Chem. Phys. 97 (8) (1992) 5876.

[17] B. Lin, M.C. Shih, T.M. Bohanon, G.E. Ice, P. Dutta, Phys. Rev. Lett. 65 (2) (1990) 191.

[18] K. Yase, S. Ogihara, M. Sano, M. Okada, J. Crystal Growth 116 (1992) 333.

[19] M. Takeuchi, S. Ueno, K. Sato, Crys. Growth Des. 3 (3) (2003) 369.

[20] S. De Jong, Triacylglycerol crystal structures and fatty acid conformations, a theoretical approach. Ph.D. Thesis, University of Utrecht, The Netherlands, 1980.

[21] K. Larsson, Physical properties - structural and physical characteristics, in: F.D. Gunstone, J.L. Harwood, F.B. Padley (Eds.), The Lipid Handbook, Chapman \& Hall, London, UK, 1986, p. 321. 